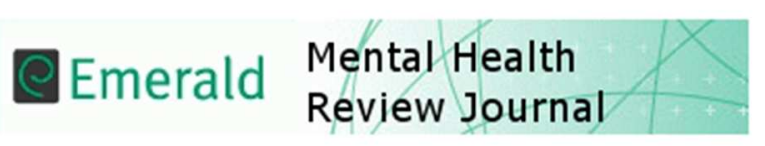

\title{
The Emotional Needs Audit (ENA): A report on its validity and reliability.
}

\begin{tabular}{|r|l|}
\hline Journal: & Mental Health Review Journal \\
\hline Manuscript ID: & MHRJ-Mar-2012-0006.R1 \\
\hline Manuscript Type: & Research Paper \\
\hline Keywords: & validity, reliability, depression, emotional distress, human givens \\
\hline \multicolumn{2}{|c}{} \\
\hline
\end{tabular}

\section{SCHOLARONE ${ }^{\text {Tx }}$ \\ Manuscripts}


Table 1. Descriptive statistics of the missing value analysis ENA items.

\begin{tabular}{lllrrrrrr}
\multirow{2}{*}{$\begin{array}{l}\text { ENA } \\
\text { Items }\end{array}$} & $\mathrm{N}$ & Mean & \multirow{2}{*}{$\begin{array}{c}\text { Std. } \\
\text { Deviation }\end{array}$} & & Count & Percent & Low & High \\
\hline Q1a & & & 2.177 & 4 & 2.3 & 0 & 0 \\
\hline Q1b & 172 & 3.74 & 2.31 & 1.771 & 47 & 26.7 & 0 & 11 \\
\hline Q1c & 167 & 3.35 & 1.873 & 9 & 5.1 & 0 & 0 \\
\hline Q2 & 170 & 3.87 & 1.987 & 6 & 3.4 & 0 & 0 \\
\hline Q3 & 172 & 4.55 & 1.974 & 4 & 2.3 & 0 & 0 \\
\hline Q4 & 173 & 2.72 & 1.686 & 3 & 1.7 & 0 & 0 \\
\hline Q5 & 173 & 2.49 & 1.627 & 3 & 1.7 & 0 & 0 \\
\hline Q6 & 174 & 4.64 & 2.185 & 2 & 1.1 & 0 & 0 \\
\hline Q7 & 174 & 4.98 & 2.420 & 2 & 1.1 & 0 & 0 \\
\hline Q8 & 170 & 4.25 & 2.636 & 6 & 3.4 & 0 & 0 \\
\hline Q9 & 172 & 4.30 & 1.997 & 4 & 2.3 & 0 & 0 \\
\hline Q10 & 170 & 3.01 & 1.815 & 6 & 3.4 & 0 & 0 \\
\hline Q11 & 171 & 2.99 & 1.966 & 5 & 2.8 & 0 & 0 \\
\hline Q12 & 172 & 2.97 & 1.851 & 4 & 2.3 & 0 & 0 \\
\hline
\end{tabular}

Table 2. Reliability analysis on ENA individual items (internal consistency - Cronbach's alpha)

No. ENA Statements

Corrected Item- Cronbach's Alpha Total Correlation if Item Deleted

Q1a Do you feel secure in your home life? 0.532

Q1b Do you feel secure in your work life? 0.345

0.842

Q1c Do you feel secure in your environment?

0.564

0.830

Q2 Do you feel you receive enough attention?

0.435

0.837

Q3 Do you give other people enough 0.280

0.846 attention?

Q4 Do you feel in control of your life most of $\quad 0.597$ the time?

Q5 Do you feel part of the wider community? 0.491

Q6 Can you obtain privacy when you need to?

0.466

0.836 


\begin{tabular}{|c|c|c|c|}
\hline Q7 & Do you have at least one close friend? & 0.194 & 0.855 \\
\hline Q8 & $\begin{array}{l}\text { Do you have an intimate relationship in } \\
\text { your life? }\end{array}$ & 0.416 & 0.842 \\
\hline Q9 & $\begin{array}{l}\text { Do you feel an emotional connection to } \\
\text { others? }\end{array}$ & 0.417 & 0.838 \\
\hline Q10 & $\begin{array}{l}\text { Do you feel you have status that is } \\
\text { acknowledged? }\end{array}$ & 0.657 & 0.825 \\
\hline Q11 & $\begin{array}{l}\text { Are you achieving things and feeling } \\
\text { competent...? }\end{array}$ & 0.670 & 0.823 \\
\hline Q12 & $\begin{array}{l}\text { Are mentally and/or physically being } \\
\text { stretched....? }\end{array}$ & 0.469 & 0.835 \\
\hline
\end{tabular}

Table 3. ENA concurrent validity.

\begin{tabular}{lllc} 
Scales & N & R & p \\
\hline ENA x SWLS & 159 & 0.62 & $<0.001$ \\
ENA x CORE-OM & 149 & -0.59 & $<0.001$ \\
(Total) & & & \\
ENA x CORE-OM & 145 & -0.51 & $<0.001$ \\
(SWB)* & & & \\
\hline
\end{tabular}

*SWB: Subjective Well-Being dimension

Table 4. ENA discriminant validity.

$\begin{array}{llll}\text { Scales } & \mathbf{N} & \mathbf{R} & \mathbf{p}\end{array}$

\begin{tabular}{lccc}
\hline ENA x CORE-OM & 145 & -0.288 & $<0.001$ \\
(Trauma) & & & \\
ENA x CORE-OM & 145 & -0.223 & $<0.01$ \\
$\begin{array}{ll}\text { Physical Problems) } \\
\text { ENA } \quad\end{array}$ & & \\
(Depression) & 159 & 0.246 & $<0.01$ \\
\hline
\end{tabular}


Table 5. ENA test-retest reliability.

1

2

3

4

5

6

7

8

9

10

11

12

13

14

15

16

17

18

19

20

21

22

23

24

25

26

27

28

29

30

31

32

33

34

35

36

37

38

39

40

41

42

43

44

45

46

47

48

49

50

51

52

53

54

55

56

57

58

59

60

\begin{tabular}{llll} 
Scales & $\mathbf{N}$ & $\mathbf{R}$ & $\mathbf{p}$ \\
\hline ENA T1 x ENA T1 & 87 & 0.468 & $<0.001$ \\
\hline
\end{tabular}




\title{
The Emotional Needs Audit (ENA): A report on its reliability and validity
}

\begin{abstract}
Purpose: To broaden the range of well-being outcomes that we can measure for patients with depressed mood and/or other mental health issues we aimed to determine the reliability and validity of a self-reported instrument that was designed by the Human Givens Institute to evaluate emotional distress (Emotional Needs Audit; ENA).

Design/Methodology: The ENA was administered to 176 patients, aged between 18 65 years (mean age: 39.2 years). The acceptability of the ENA was examined as well as its internal consistency (Chronbach's alphas). ENA was administered at four time points and test-retest reliability was conducted between times 1 and 2 . The data from three scales also administered to these patients (SWLS, CORE-OM and HADS) were used to aid the conduct of the ENA construct validity (concurrent and discriminant). Analysis of the ENA sensitivity/specificity was also performed.

Findings

ENA items in the majority (except one) were shown to have good acceptability. The internal consistency was also very strong (Cronbach-alpha: 0.84) ENA construct validity also revealed positive results for the ENA: concurrent validity $(r=0.51-$ $0.62 ; \mathrm{p}<.001)$; discriminant validity $(\mathrm{r}=0.22-0.28 ; \mathrm{p}<.01)$. Test-retest reliability was $\mathrm{r}=0.46(\mathrm{p}<.001)$. Finally, ENA demonstrated high sensitivity $(80 \%)$, and moderate specificity $(35 \%)$.

Originality/Value
\end{abstract}

ENA was shown to be a valid and reliable instrument for measuring wellbeing, quality of life and emotional distress. It also allows insight into the causes of 
1

2

3

4

5

6

7

8

10

11

12

13

14

15

16

17

18

19

20

21

22

23

24

25

26

27

28

29

30

31

32

33

34

35

36

37

38

39

40

41

42

43

44

45

46

47

48

49

50

51

52

53

54

55

56

57

58

59

60

symptoms, dissatisfaction and distress. It is suggested that this tool has complementarity to standardised tools when used in clinical practice.

Article Classification: Research Paper

Keywords: depression, emotional distress, human givens, reliability, validity 


\section{Background}

In 2007, Sandwell Primary Care Trust funded a quasi-experimental research project (nonequivalent groups design) to test the effectiveness of therapy based on the Human Givens approach in the treatment of depression in primary care. The experimental design was designed to compare a talking treatment based on the Human Givens approach against standard treatment.

The basic principle of the Human Givens approach to therapy is that we are all born with vital physical and emotional needs along with the innate resources to help us fulfil such needs. These needs and resources together make up the 'givens' of human nature and are the means via which individuals can reach their genetic human potential. When these emotional needs are not met, or when the innate resources are used incorrectly, individuals may experience mental distress (Griffin and Tyrrell, 2004). As an approach to therapy, Human Givens employs a wide range of techniques adopted from other approaches, such as Cognitive Behavioural Therapy (CBT), Solution Focused Therapy (SFT), NeuroLinguistic Programming (NLP) and other therapeutic models, such as the rewind technique.

The ENA was developed by the Human Givens Institute (HGI, 2006), particularly the founders of the Human Givens (Griffin and Tyrrell) to assess emotional distress. Their premise for the development of ENA was that when a person is getting their innate physical and emotional needs met in a balanced way they will be mentally healthy (unless they are also traumatised or brain damaged in some way). The Emotional Needs Audit is a simple diagnostic self-report questionnaire which claims to have the ability to identify where potential problems in someone's life might lie, and which particular inherent needs are not fulfilled. 
The study focused on service users who were screened with moderate to severe depressed mood, as identified by their General Practitioner (GP) or Practice Nurse.

Participating GP practices were designated as either 'Human Givens' practices able to refer patients screened with depressed mood to Human Givens service, or 'Control' practices offering standard services only.

In the present paper we have used the data collected to run a series of statistical tests to evaluate the reliability and validity of the ENA in comparison of other established well being outcome measures.

\section{Methods}

\section{Participants and data collection}

A quasi experimental design study of patients receiving treatment for depression in Sandwell Primary Care was conducted from June 2007 to January 2011. The present paper is part of this larger project which was conducted to determine the effectiveness of the Human Givens therapy to mental health in Sandwell and its methodology and results are published elsewhere, (Staffordshire University, 2011). Only the methodology that relates to the present paper is described here.

Patients were eligible to participate if they were: a) at least 18 years of age; b) scored 11 or above on the Hospital Anxiety and Depression Scale (HADS) (Zigmond \& Snaith 1983) on the depression element, c) able to read and understand English; and d) provided written consent. Ethical approval was obtained from the Sandwell and West Birmingham Research Ethics Committee. 
Two weeks after being screened for depression by their General Practitioner, patients who agreed to participate were asked to complete three self administered questionnaires regarding their well being. These were the Satisfaction with Life Scale (SWLS; Diener et al 1985), the Clinical Outcomes in Routine Evaluation Outcome Measure (CORE-OM; Barkham 1998) and Emotional Needs Audit (ENA; HGI 2006). This procedure was repeated at 4, 8 and 12 months following the date of treatment referral. Additional data on demographic and socioeconomic variables were also collected.

\section{Validation Measures \\ Emotional Needs Audit}

The Emotional Needs Audit (ENA; HGI, 2006): is a 14-item questionnaire developed by the Human Givens Institute (HGI) which aims to identify where the potential problems and distress in someone's life might be located. The stated basis of Human Givens therapy (Griffin \& Tyrell 2004) is that well being and quality of life is related to whether the individual's needs are being met and the ENA is claimed to be a means to identify the areas that cause the problems. Thus, there is a difference with the other instruments, which focus on the results of ill-health rather than the causes. The areas covered are: security, attention, control, part of wider community, privacy, intimate connection to others, status, competency and emotional \& physical stretching. ENA consists of 12 items, and the first item is divided into three subsections (a, b, and c). Each item of the ENA is measured using a Likert 7-point scale ranging from 1 (No; i.e. need is unfulfilled) to 7 (Yes; i.e. need is fulfilled); the middle score indicates "sometimes". The total ENA score can be obtained by summing the 12 items and ranges between 14 and 98 . High scores indicate better quality of life and well being, with needs being met. Scores below 3 in individual items indicate serious lack of fulfilment in that particular need (HGI 2006). The ENA form takes about five minutes to complete. 


\section{Satisfaction with Life Scale}

The Satisfaction with Life Scale (Diener et al 1985): SWLS is a standardised and widely used self-report instrument. It consists of 5-items that assess an individual's global judgement of life satisfaction. Each item of the SWLS is measured using a Likert 7-point scale ranging from 1 (strongly disagree) to 7 (strongly agree); the middle score indicates a neutral position. The total SWLS score can be obtained by summing the 5 items and ranges between 5 and 35 . The form takes about one minute to complete.

\section{Clinical Outcomes in Routine Evaluation - Outcome Measure (CORE-OM: NHS Standard)} CORE-OM (Barkham et al 1998; Evans et al 2000; 2002). This is a standardised self-report questionnaire to assess efficacy and effectiveness in psychological treatments. It consists of 34 items covering four domains: subjective well-being (4 items), symptoms (12 items), functioning (12 items) and risk (6 items). Within the symptoms domain subscales address anxiety (4 items), depression (4 items), physical problems (2 items) and trauma (2 items). The functioning domain has subscales for general functioning (4 items), close relationships (4 items) and social relationships (4 items). Risk has subscales risk to others (2 items) and risk to self ( 4 items). Items are scored on a five-point scale from 0 ("not at all") to 4 ("all the time"). Half of the items focus on low-intensity problems (e.g. "I feel anxious/nervous") and half focus on high-intensity problems (e.g. "I feel panic/terror"). Eight items are keyed positively. This questionnaire takes approximately 10-15 minutes to complete. Since its development, CORE-OM has been validated with samples from the general population, NHS primary and secondary care, and in older adults. 


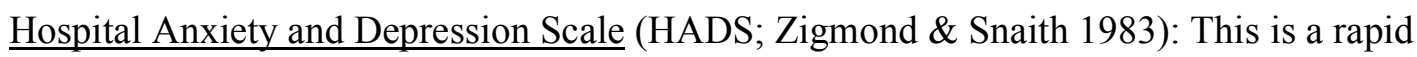
self-report questionnaire which measures depression and generalised anxiety. It is equally useful in hospital, out-patient and community settings. HADS allows the presence and severity of both anxiety and depression to be established simultaneously. It consists of 14 items each rated from 0 to 3 according to severity of difficulty experienced.

Six items require reverse scoring, after which depression (HADS-D $=7$ items) and anxiety (HADS-A $=7$ items) subscale totals can be summed. Each subscale score can range from 0 to 21. The scores can then be interpreted as indicating mild (8-10), moderate (11-15), or severe (>16) difficulty (Snaith \& Zigmond, 1994), and a mood disorder considered highly likely with a score of 11 or over (Snaith 2003).

\section{Results}

\section{Sample demographic information and descriptive statistics}

Sample size: A total of 379 patients met all of the inclusion criteria, out of which $176(46.5 \%)$ patients agreed to participate forming the study population.

Age: The mean age of the participants was 39.2 years (SD: 11.83) with minimum and maximum age scores of 18 and 65 years respectively.

Ethnicity: Eighty-three point eight percent $(83.8 \%)$ of participants identified themselves as white British whilst the remaining 16.2\% were from African, Caribbean, Asian or mixed background. Sixty two point two percent (62.2\%) were females and $31.4 \%$ males $(6.4 \%$ missing). This is consistent with the reports that less males tend to report depression than females (Kessler et al, 2003, Piccinelli \& Wilkinson 2000, Nolen-Hoeksema, 2002). 
Depression history: Forty four point nine percent $(44.9 \%$ had no previous history of depression, whilst $35.7 \%$ had a past history; the remaining sample gave no information.

Descriptive statistics for ENA, SWLS and CORE-OM: The mean scores for ENA and SWLS were 3.9 (SD: 1.23) and 2.4 (SD: 1.19) respectively in a scale 1-7 (where the lower score the lower the well being). The mean score for CORE-OM (Subjective Well-being subscale) was 2.8 (SD: 0.74) in a subscale 1-5 (where the higher the score the lower the well being).

\section{ENA Reliability and Validity tests}

The ENA acceptability, internal consistency, test-retest reliability and construct validity were examined. The ENA sensitivity and specificity were also tested. All statistical analysis was conducted using SPSS 17 for Windows (SPSS 2008). Results are presented and discussed below.

\section{ENA Acceptability}

Acceptability was evaluated by examining completion rates for individual questions and overall questionnaire. Acceptability was also assessed during the participant interviews by asking participants how they felt about the experience of completing the questionnaire, and whether they understood all the questions. The most essential prerequisites of any psychological measurement tool are that respondents are able to understand it and are also prepared to complete it. This concept of acceptability has been used by other published research studies (Tarrant et al, 2009, Brédart et al, 2002).

As a result, of those who participated in the study, $156(89.7 \%)$ fully completed the ENA questionnaire at Time 1, and 87 out of 89 at Time $2(97.7 \%)$. Missing value analysis was also 
conducted indicating missing data for individual questions ranging from $1.1 \%$ to $5.1 \%$. This was the case for all ENA items except one. The only item with a high missing data value was $1 \mathrm{~b}$ (Do you feel secure in your work life?) with an omission rate of $26.7 \%$ (47 omissions). This was felt to be attributable to the high level of unemployment within Sandwell's population. Descriptive statistics of the missing value analysis items are shown in Table 1.

The normality of the distribution of the responses as part of the questionnaire acceptability has also been recommended by Tarrant et al (2009). However, this was not included in the present analysis as the responses were expected to be skewed and non-normal due to the nature of the study (i.e. measuring emotional needs in a depressed population).

On the whole, participants reported positive experiences and good understanding of the ENA questions, with mean overall scores ranging from 2.31 to 4.98 across the items.

Table 1 goes here

\section{ENA Internal Consistency}

Internal reliability is indicated by Cronbach's coefficient $\alpha$ (Cronbach 1951) which specifies the proportion of the variance that is covariant between items. The values of $\alpha$ range between 0 (weak reliability) and 1 (perfect reliability). Values of $\geq 0.7$ indicated acceptable internal consistency for research purposes. Very high values $(>0.9)$ suggest that too many items are being used, or that items are of similar meaning - i.e. not adding any new information to the scale (George \& Mallery 2003; Clark-Carter 1997). Corrected item-total correlations should be $>0.4$ (Gliem \& Gliem 2003). Table 2 shows that 11 out of 14 ENA items $(78.6 \%$ ) had corrected-item total correlations of $>0.40$. Moreover, the overall value of $\alpha$ would not reduce below 0.8 if any item was deleted. 
Table 2 goes here

The overall value of $\alpha$ was 0.84 , suggesting that the items of the ENA had high internal consistency.

\section{ENA Validity analysis}

The construct validity of the ENA was tested, including the domains of concurrent (convergent) and discriminant (divergent) validity.

Concurrent (convergent) validity. This test measures how similar the two constructs (i.e. two questionnaires) are. The stronger the relationship between the two constructs, the higher the concurrent validity. Failure to correlate with appropriate specific measures would suggest invalidity. Pearson's correlations (Table 3) were conducted between ENA, SWLS and CORE-OM (Total and Subjective Well-Being dimension) at Time 1. The correlation coefficients were moderate or strong (Swinscow \& Campbell 2002), suggesting a good concurrent validity for ENA.

Table 3 goes here

Discriminant (divergent) validity._This test measures whether items that should not be related are in reality not related. If we have discriminant validity, the relationship (i.e. Pearson's r) between measures from different constructs should be very low. Pearson's correlation (see Table 4) was calculated between ENA and CORE-OM (Trauma and Physical Problems dimensions) as well as between ENA and HADS (Depression) at Time 1, all of which are 
dissimilar constructs to that of ENA which assesses emotional needs. The very low correlation coefficients between ENA and the three other measures suggests good discriminant validity for ENA.

Table 4 goes here

\section{ENA Test-Retest Reliability}

This kind of reliability is used to assess the consistency of an instrument across time. Testretest reliability is measured by administering a test twice at two different points in time. This type of reliability assumes that there will be no substantial change in the quality or construct being measured. However, in the present study, ENA is a measure that was administered at different time points anticipating a degree of improvement in the well-being of the participants.

Pearson's correlations were calculated between ENA scores at time 1 (prior to treatment) and ENA at time 2 (4 months after treatment). The results revealed a moderate significant relationship between ENA at Times 1 and $2(\mathrm{r}=0.46, \mathrm{p}<.001, \mathrm{n}=87)$ (Table 5), thus indicating satisfactory test-retest reliability for the ENA.

Table 5 goes here

\section{ENA Sensitivity and Specificity analysis}

Wellbeing detection was scored and evaluated on the basis of the SWLS. A (SWLS) score less than 4 is considered to indicate low emotional well being (Diener et al 1985). In order to determine the sensitivity and specificity, ENA was tested against the SWLS outcomes. The Receiver Operating Characteristics (ROC) curve analysis was calculated to determine the 
sensitivity, specificity and area under ROC curve of the ENA. The sensitivity of a test refers to how many real cases of a condition a particular test can accurately find. A very sensitive test is likely to give a relatively high number of false-positive results, but few true positives will be missed. The specificity of a test is its ability to correctly designate individuals who do not have a condition as negative. A highly specific test would yield few false positive results. The area under a ROC curve quantifies the overall ability of the test to discriminate between those individuals with a condition and those without a condition. A very inaccurate test has an area of 0.5, while a perfect test has an area of 1.00 (Hanley \& McNeil 1982).

Figure 1. Sensitivity and specificity of ENA

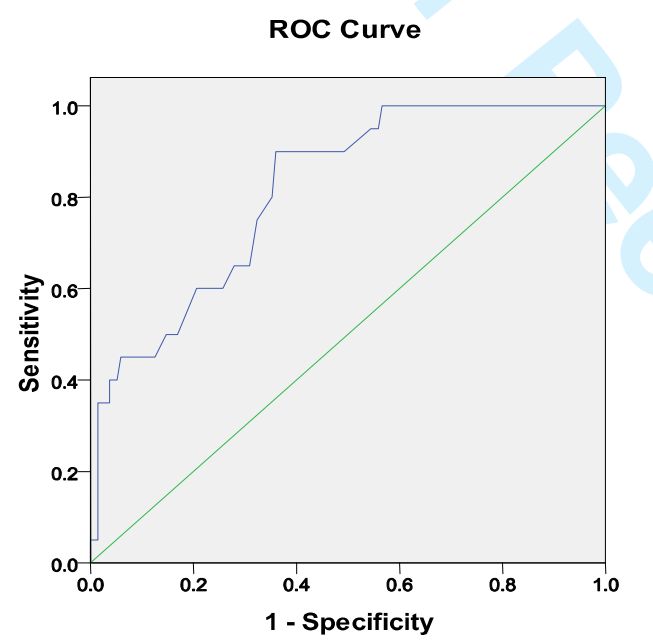

The results at Figure 1 show that ENA had a very high sensitivity of $80 \%$ and low to moderate specificity at $35 \%$. These results indicate that ENA will give an accurate assessment of positive results, but may yield a low number of false positive results. The area under the (ROC) curve value was considered "good" at 0.81 , indicating that ENA is capable of accurately detecting people with low emotional well being. Clinicians will need to take into account the low to moderate specificity of the ENA. Many patients will have some areas in their lives that may be being compromised by their current circumstances or that they might or might not wish to work upon to improve, which the ENA may highlight, but this is a grey area that the clinician will need to further explore with their patient. We would also 
consider the use of ENA as only part of a consultation process that would also encapsulate clinical experience and investigation to avoid the dangers of false positive results.

\section{Discussion}

The correlation results of the ENA to already validated/standardised wellbeing and depression scales suggests that quality of life and mental ill-health and well-being is proportionally related to how well we are able to meet our emotional needs. This supports the Human Givens underlying principles and view that getting those needs met should be the goal of therapy. Unmet emotional needs can be identified using the ENA and further, effectiveness of treatment may be monitored by completing the ENA before and after sessions, alongside other previously validated measures.

Our findings show that the Emotional Needs Audit appears to be acceptable in the domains of internal consistency, test-retest reliability, construct validity, and sensitivity, with a "good" ROC value. This suggests that it is a valid and reliable instrument for measuring wellbeing, quality of life and emotional distress.

While the ENA seems to be able to measure similar domains to the SWLS and CORE-OM effectively, we suggest that the ENA scale has additional advantages. Firstly, the CORE-OM measures symptoms and the SWLS measures overall satisfaction with life. Neither scale, however, offers insights into the causes of symptoms or causes of dissatisfaction and distress. Our observations suggest that when faced with a patient in distress, it is necessary to evaluate not only the level of distress but also the causes of distress. The ENA allows the practitioner to evaluate such causes. Indeed, it allows the practitioner to focus in the following areas: 
Security, Attention (to give and receive it), Sense of autonomy and control, Emotional intimacy, Feeling part of a wider community, Privacy, Sense of status within social groupings, Sense of competence and achievement, Meaning and purpose .

Although there were differences between the individual focus of the instruments used in this evaluation, all were measures of wellbeing. The SWLS and ENA correlated most closely, low life satisfaction (SWLS) would correspond with low emotional needs (ENA). The CORE$\mathrm{OM}$ on the other hand, is a more clinically oriented instrument, and is multidimensional. Its inclusion in the study was justified by its ability to relate to both clinical and social/emotional aspects.

\section{Conclusion}

We conclude that in addition to measuring symptoms and satisfaction with life, the ENA is capable of providing understanding of the causes of any problems, and therefore has the potential to be a useful instrument in clinical practice. Indeed we would argue that the results from ENA might allow a practitioner to develop a level of communication that might therapeutically assist the start of treatment. 


\section{References}

Barkham, M., Evans, C., Margison, F., McGrath, G., Mellor-Clark, J., Milne, D. and Connell, J. (1998), "The rationale for developing and implementing core outcome batteries for routine use in service settings and psychotherapy outcome research", Journal of Mental Health, Vol. 7, No. 1, pp. 35-47.

Brédart. A., Razavi, D., Robertson, C., Brignone, S., Fonzo, D., Petit, J.Y. and de Haes, J.C. (2002), "Timing of patient satisfaction assessment: effect on questionnaire acceptability, completeness of data, reliability and variability of scores", Patient Education and Counselling, Vol. 46, No. 2, pp. 131-136.

Clark-Carter, D. (1997), Doing Quantitative Psychological Research: From Design to Report, Hove, Psychology Press.

Cronbach, L.J. (1951), "Coefficient alpha and the internal structure of tests", Psychometrika, Vol. 16, No. 3, pp.297-334.

Diener, E., Emmons, R. A., Larsen, R. J., \& Griffin, S. (1985), “The Satisfaction with Life Scale", Journal of Personality Assessment, Vol. 49, pp. 71-75.

Evans, C., Connell, J., Barkham, M., Margison, F., McGrath, G., Mellor-Clark, J. and Audin, K. (2002), "Towards a standardised brief outcome measure: psychometric properties and utility”, British Journal of Psychiatry, Vol. 180, No. 1, pp. 51-60. 
Evans, C., Mellor-Clark, J., Margison, F., Barkham, M., Audin, K., Connell, J. and McGrath, G. (2000), "CORE: Clinical Outcomes in Routine Evaluation”. Journal of Mental Health, Vol. 9, No. 3, pp. 247-255.

George, G, and Mallery, P. (2003), SPSS for windows step by step: A simple guide and reference, 11.0 update. Boston, MA, Allyn \& Bacon.

Gliem, J.A., and Gliem, R.R. (2003), Calculating, Interpreting, and Reporting

Cronbach's Alpha Reliability Coefficient for Likert-Type Scales”, Paper presented at Midwest Research to Practice Conference in Adult, Continuing, and Community Education, October 8th, 2003, The Ohio State University, Columbus, OH, available at: https://scholarworks.iupui.edu/bitstream/handle/1805/344/Gliem\%20\&\%20Gliem.pdf (accessed 7 March 2012).

Griffin, J. and Tyrrell, I. (2004), Human Givens: A new approach to emotional health and clear thinking, Chalvington, HG Publishing.

Hanley, J. A., and McNeil, B.J. (1982), “The meaning and use of the area under a receiveroperating characteristic (ROC) curve". Radiology, Vol. 143, pp. 29-36.

Kessler, R.C., Berglund, P., Demler, O., Jin, R., Koretz, D., Merikangas, K.R., Rush, A.J., Walters, E.E., Wang, P.S.(2003), “The epidemiology of major depressive disorder: results from the National Comorbidity Survey Replication (NCS-R)", Journal of the American Medical Association, Vol. 289, No. 23, pp. 3095-105. 
Nolen-Hoeksema, S. (2002), “Gender differences in depression”, Current Directions in Psychological Science, Vol. 10, No. 5, pp. 173-6.

Piccinelli, M., Wilkinson, G. (2000), “Gender differences in depression. Critical review”, British Journal of Psychiatry, Vol. 177, pp. 486-92.

SPSS for Windows, Rel. 17.0.0. (2008). Chicago: SPSS Inc.

Corp, N., Tsaroucha, A. and Kingston, P. (2011), “A Health Impact Assessment of Reconfigured Mental Health Services in Sandwell Primary Care Trusts", working paper, Staffordshire University, Stafford, 2011.

Snaith, R.P. (2003), “The Hospital Anxiety And Depression Scale”, Health and Quality of Life Outcomes, Vol. 1, No. 29.

Snaith, R.P., and Zigmond, A.S. (1994), HADS: Hospital Anxiety and Depression Scale, Windsor, NFER Nelson.

Swinscow, T.D.V, and Campbell, M.J. (2002), Statistics at square one, London, BMJ Books.

The Human Givens Institute (HGI) (2006), “The Emotional Needs Audit (ENA)”, available at: http://www.hgi.org.uk/ena/, (accessed 6 May 2010).

Tarrant, C., Baker, R., Colman, A.M., Sinfield, P., Agarwal, S., Mellon, J.K., Steward, W. and Kockelbergh, R. (2009), “The Prostate Care Questionnaire for Patients (PCQ-P): 
Reliability, validity and acceptability”, BMC Health Services Research, Vol. 9, No. 199 doi:10.1186/1472-6963-9-199

Zigmond, A.S., and Snaith, R.P. (1983), “The Hospital Anxiety and Depression Scale” Acta Psychiatrica Scandinavica, Vol. 67, pp. 361-70. 